\title{
Probing the Constituent of QCD Plasma in CUJET3.1/CIBJET Framework
}

\section{Shuzhe Shi*}

Department of Physics, McGill University, Montreal, Quebec H3A 1J3, Canada

Center for Exploration of Energy and Matter, Indiana University, Bloomington, IN 47408, USA

E-mail: shuzhe.shiephysics.mcqill.ca

\section{Jinfeng Liao}

Physics Department and Center for Exploration of Energy and Matter, Indiana University,

2401 N Milo B. Sampson Lane, Bloomington, IN 47408, USA

E-mail: Liaojidindiana.edu

\section{Miklos Gyulassy}

Nuclear Science Division, Lawrence Berkeley National Laboratory, Berkeley, CA 94720, USA

MTA Wigner Research Centre for Physics, 1525 Budapest, Hungary

Institute of Particle Physics, Central China Normal University, Wuhan, China

Pupin Lab MS-5202, Department of Physics, Columbia University, New York, NY 10027, USA

E-mail: ma150ecolumbia.edu

In this proceeding we report results of a comprehensive global analysis of ultra-relativistic heavyion collision data using the updated CUJET3.1 framework. By comparing CUJET results with (CUJET3.1) versus without (CUJET2.0) chromo-magnetic monopole $(\mathrm{cmm})$ degrees of freedom, we find that the cmm scenario is more favored by experimental data. Finally, we use the event-byevent generalization of the CUJET framework, CIBJET, and show that fluctuations in the initial conditions do not significantly alter our conclusions for $R_{A A}$ and $v_{2}$.

13th International Workshop in High pT Physics in the RHIC and LHC Era (High-pT2019)

19-22 March 2019

Knoxville, Tennessee, USA

\footnotetext{
*Speaker.
} 


\section{Introduction}

High energy quark and gluon jets, initially generated in rare perturbative QCD processes, lose energy and diffuse transversely along their paths due to interactions with microscopic constituents of the hot quark-gluon plasma created by heavy ion collisions. Such high $p_{T}$ processes provide an independent probe of the evolution history of the QCD matter produced in such collisions. Recent high-precision data from $\mathrm{LHC} \mathrm{Pb}+\mathrm{Pb}$ collisions on jet quenching and azimuthal asymmetry observables over wide kinematics and centrality ranges provide an opportunity to quantitatively constrain and differentiate competing models of jet-medium interactions, as well as varied assumptions of the chromo-electric and magnetic field structure of the bulk QCD "perfect fluids" produced in ultra-relativistic nuclear collisions.

In this work we utilize the CUJET3 model, a jet energy loss simulation framework, to probe the color structure of semi-quark-gluon-monopole plasma (sQGMP), which integrates two essential elements of confinement, i.e. the suppression of quarks/gluons and emergent magnetic monopoles. The CUJET3 model employs the TG elastic energy loss formula [四] for collisional processes, and the dynamical DGLV opacity expansion theory []] with the Liao-Shuryak chromo-magneticmonopole scenario [B] for radiational processes. Detailed description can be found in previous

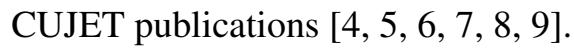

\section{Parameter Calibration}

There are two key parameters in the CUJET3 model. One is $\alpha_{c}$, the value of QCD running coupling at the non-perturbative scale $Q^{2}=T_{c}^{2}$. It sensitively influences the overall opaqueness of the hot medium. The other is $c_{m}$, being the coefficient to scale the magnetic screening mass in the medium $c_{m}=\mu_{M} /(g \mu)$. It influences the contribution of the magnetic component to the jet energy loss. The increase of $c_{m}$ leads to the enhancement of monopole mass, hence overall opaqueness.

To constrain these two parameters, we compare the results from CUJET3.1 calculations with high $p_{T}$ experimental data of both $R_{A A}$ and $v_{2}$, for central $(0-5 \%, 0-10 \%)$ and semi-central $(10-30 \%, 20-30 \%)$ heavy-ion collisions at beam energy $\sqrt{s_{N N}}=0.2,2.76$ and $5.02 \mathrm{TeV}$ [س0],

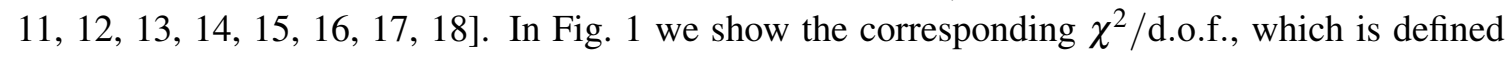
as the ratio of the squared difference between the experimental data points and corresponding CUJET3 expectation, to the quadratic sum of experimental statistic and systematic uncertainties for that data point. With the smallest $\chi^{2} /$ d.o.f. closest to unity, we obtain the optimal parameter set as $\alpha_{c}=0.9 \pm 0.1$ and $c_{m}=0.25 \pm 0.03$.

With taking the optimal parameter, we show in Fig. $\square$ the comparison with the experimental data. While both sQGMP schemes ( $\chi_{T}^{L}$ and $\chi_{T}^{u}$, see e.g. [ $[\mathbb{b}]$ for more detail) give similar jet quenching variables, the QGP scheme gives similar $R_{A A}$ but less azimuthal anisotropy. In particular, one can see clearly from the quantitative value of their $\chi^{2} /$ d.o.f. that the theoretical expectations of both sQGMP schemes are in good consistency with the experimental data, and that of the QGP scheme, without $\mathrm{cmm}$ degree of freedom, differs significantly from the highly precise LHC $v_{2}$ measurements. The $\chi^{2}$ analysis strongly supports the need of $\mathrm{cmm}$ degrees of freedom, but remains robust on the specific quark liberation scheme. 

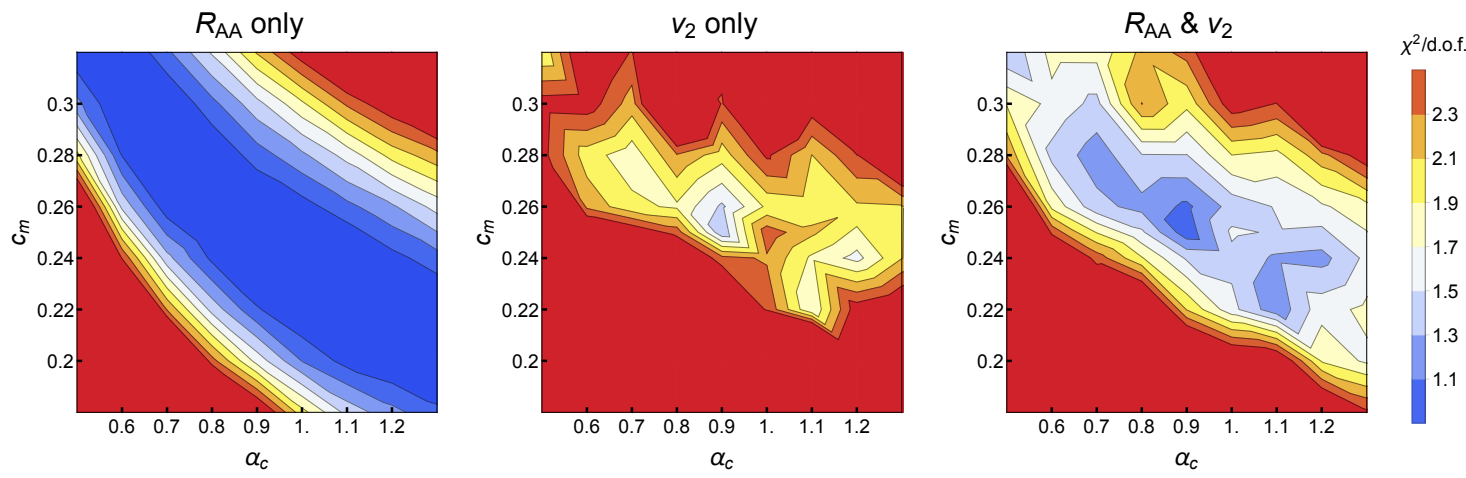

Figure 1: $\chi^{2} /$ d.o.f. comparing CUJET3.1 results with RHIC and LHC data. Left: $\chi^{2} /$ d.o.f. for $R_{A A}$ only. Middle: $\chi^{2} /$ d.o.f. for $v_{2}$ only. Right: $\chi^{2} /$ d.o.f. including both $R_{A A}$ and $v_{2}$.
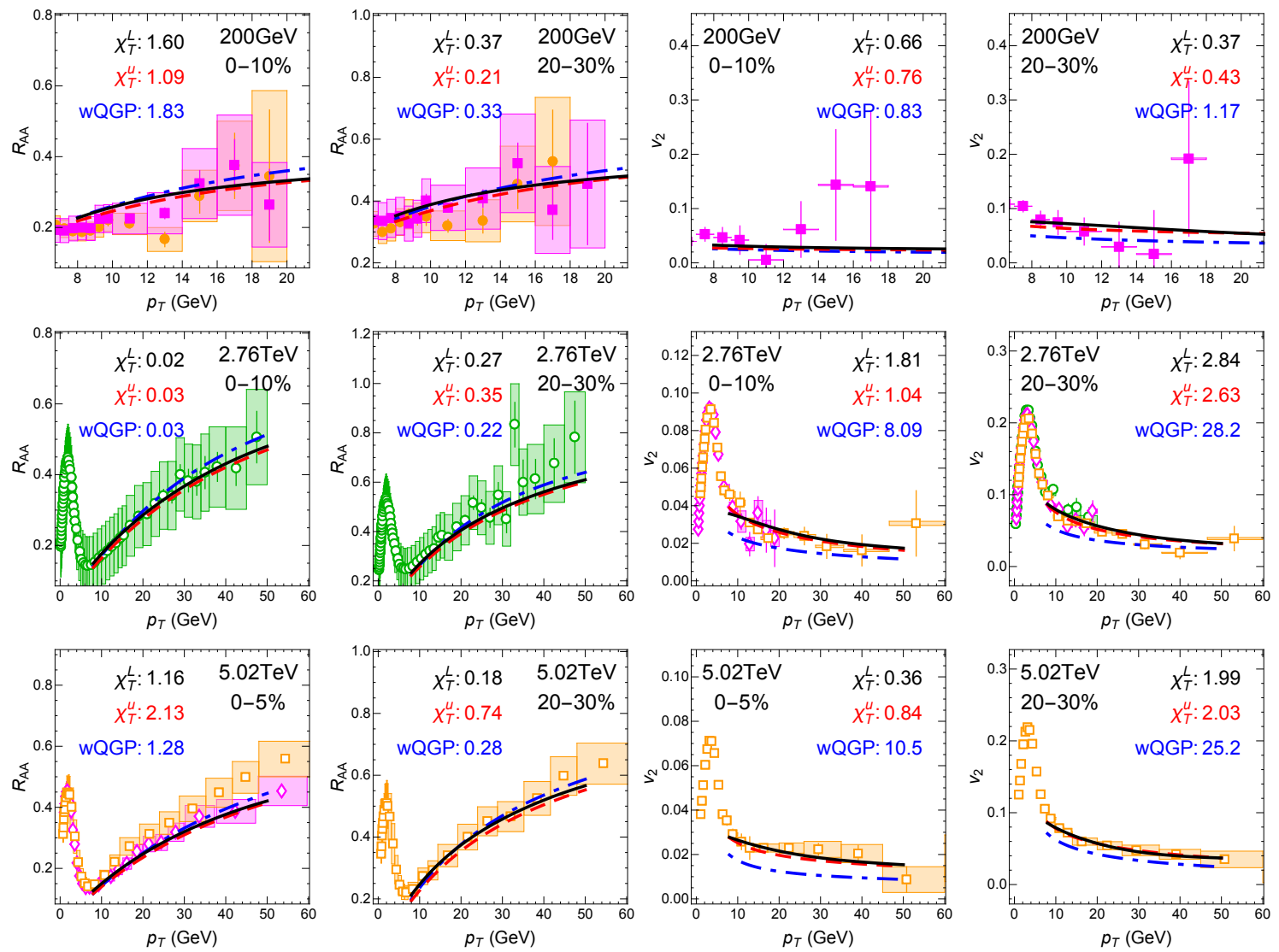

Figure 2: CUJET theoretical expectation of light hadron $R_{A A}$ and $v_{2}$ using three different schemes: sQGMP $\chi_{T}^{L}$-scheme (black solid), sQGMP $\chi_{T}^{u}$-scheme (red dashed), wQGP/CUJET2 scheme (blue dashed dotted). Corresponding $\chi^{2} /$ d.o.f. are shown, with respect to following experimental data: PHENIX 2008 (orange

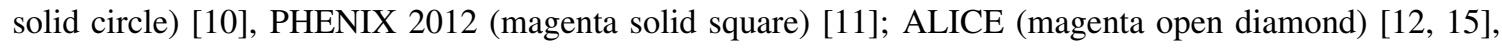
ATLAS (green open circle) [ए3, ए6], CMS (orange open square) [ए4, ㅁ], ए8].

\section{Influence of Soft-Hard Correlation}

Some recent research [2]] points out that the experimental measurements of hard particle har- 


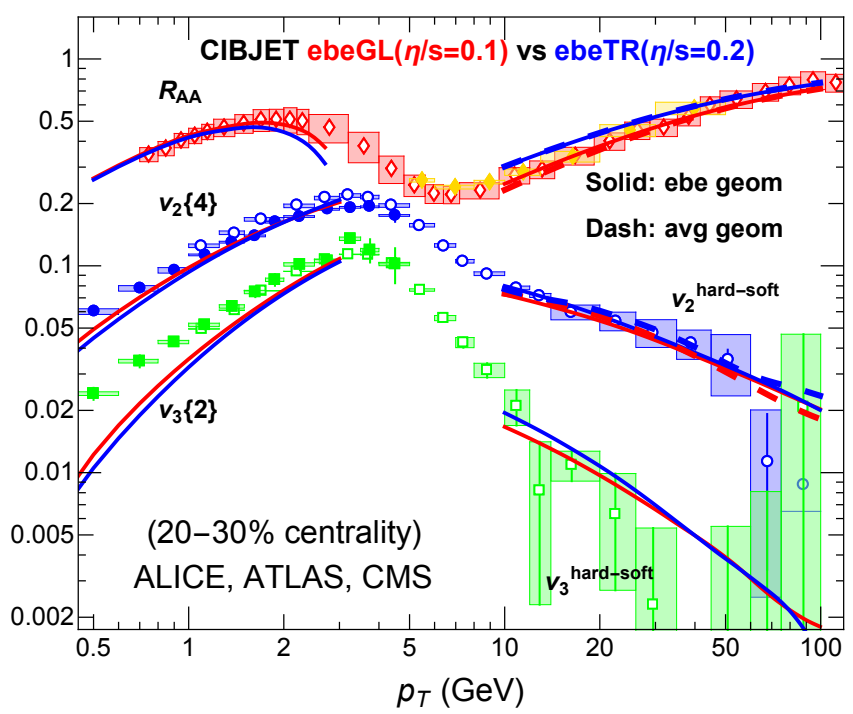

Figure 3: Nuclear modification factor $R_{A A}$ as well as the second and third harmonic coefficients $v_{2}$ and $v_{3}$ of final hadron azimuthal distribution as functions of $p_{T}$ for $20-30 \% \mathrm{~Pb}+\mathrm{Pb}$ collisions at 5.02 ATeV. Solid curves are obtained from ebe calculations, while the dashed curves depict averaged smooth geometry. CIBJET results in both soft and hard regions, with either MonteCarlo Glauber (red) or Trento (blue) initial conditions, are in good agreement with experimental data from ALICE, ATLAS, and CMS [ㅁ, ㅁ], ㅁ], ㅁ] [2]].

monics $v_{n}^{f}$ are performed with respect to event-wise soft harmonics, and event-by-event (ebe) fluctuations of the bulk initial condition may play an important role. Within the CUJET3 framework, we investigated the influence of ebe fluctuating hydro initial conditions with a generalized CIBJET framework, with the results reported in Ref. [8, Q 9 ]. In Fig. B], we present the CIBJET results of $R_{A A}, v_{2}$ and $v_{3}$ for centrality of 20-30\%, which show excellent agreement with experimental data and demonstrate the correct centrality dependence of the CIBJET results. One conclusion found with CIBJET is that the $p_{T}$ and centrality dependence of the elliptic $v_{2}^{f}\left(p_{T}, C\right)$ azimuthal harmonics shows quantitative consistency at a $\sim 10 \%$ level between calculations with averaged smooth bulk geometry and those with fluctuating initial conditions. This conclusion is true for a variety of centrality class, while different from the ebeIC + vUSPhydro + BBMG framework in Ref. [R]], which found a much larger sensitivity (factor $\sim 2$ ) of the hard elliptic harmonic to ebe fluctuations. The CIBJET computation justifies the use of averaged smooth geometry in the CUJET3 framework.

\section{Summary and Outlook}

To summarize, we report results of a comprehensive global analysis of nuclear collision data using the updated CUJET3.1 framework. By comparing CUJET results with wQGP versus sQGMP scenarios, we find that RHIC and LHC experimental data favor the existence of chromo-magnetic monopole. We further find that our conclusions for $R_{A A}$ and $v_{2}$ are insensitive to fluctuation of hydro initial conditions. We end by discussing our expectation for di-jet measurements. Di-jet accoplanarity and imbalance observables are sensitive to different jet path-length and temperature dependence, and are biased to different geometric distribution of initial jet creation spots. Consequently, we expect that future precise measurements could offer another opportunity to distinguish color constituent models. Some of the CUJET preliminary results have been reported in Ref. [22], and more detailed, systematic theoretical predictions will be presented in the near future. 


\section{Acknowledgments}

The authors are particularly grateful to Dr. Jiechen Xu for major contributions in establishing the CUJET3 framework. MG acknowledges support from IOPP of CCNU, Wuhan China. JL is partly supported by the National Science Foundation under Grant No. PHY-1913729. SS is supported by the Natural Sciences and Engineering Research Council of Canada. JL and SS are also grateful to the support by the National Science Foundation under Grant No. PHY-1352368.

\section{References}

[1] M. H. Thoma and M. Gyulassy, Nucl. Phys. B 351, 491 (1991).

[2] M. Gyulassy, P. Levai and I. Vitev, Nucl. Phys. B 594, 371 (2001).

[3] J. Liao and E. Shuryak, Phys. Rev. Lett. 101, 162302 (2008) doi:10.1103/PhysRevLett.101.162302 [arXiv:0804.0255 [hep-ph]].

[4] A. Buzzatti and M. Gyulassy, Phys. Rev. Lett. 108, 022301 (2012) doi:10.1103/PhysRevLett.108.022301 [arXiv:1106.3061 [hep-ph]].

[5] J. Xu, A. Buzzatti and M. Gyulassy, JHEP 1408, 063 (2014) doi:10.1007/JHEP08(2014)063 [arXiv:1402.2956 [hep-ph]].

[6] J. Xu, J. Liao and M. Gyulassy, Chin. Phys. Lett. 32, no. 9, 092501 (2015) doi:10.1088/0256-307X/32/9/092501 [arXiv:1411.3673 [hep-ph]].

[7] J. Xu, J. Liao and M. Gyulassy, JHEP 1602, 169 (2016) doi:10.1007/JHEP02(2016)169 [arXiv:1508.00552 [hep-ph]].

[8] S. Shi, J. Liao and M. Gyulassy, Chin. Phys. C 42, no. 10, 104104 (2018) doi:10.1088/1674-1137/42/10/104104 [arXiv:1804.01915 [hep-ph]].

[9] S. Shi, J. Liao and M. Gyulassy, Chin. Phys. C 43, no. 4, 044101 (2019) doi:10.1088/1674-1137/43/4/044101 [arXiv:1808.05461 [hep-ph]].

[10] A. Adare et al. [PHENIX Collaboration], Phys. Rev. Lett. 101, 232301 (2008) doi:10.1103/PhysRevLett.101.232301 [arXiv:0801.4020 [nucl-ex]].

[11] A. Adare et al. [PHENIX Collaboration], Phys. Rev. C 87, no. 3, 034911 (2013) doi:10.1103/PhysRevC.87.034911 [arXiv:1208.2254 [nucl-ex]].

[12] B. Abelev et al. [ALICE Collaboration], Phys. Lett. B 720, 52 (2013) doi:10.1016/j.physletb.2013.01.051 [arXiv:1208.2711 [hep-ex]].

[13] G. Aad et al. [ATLAS Collaboration], Phys. Lett. B 707, 330 (2012) doi:10.1016/j.physletb.2011.12.056 [arXiv:1108.6018 [hep-ex]].

[14] S. Chatrchyan et al. [CMS Collaboration], Phys. Rev. Lett. 109, 022301 (2012) doi:10.1103/PhysRevLett.109.022301 [arXiv:1204.1850 [nucl-ex]].

[15] B. Abelev et al. [ALICE Collaboration], Phys. Lett. B 719, 18 (2013) doi:10.1016/j.physletb.2012.12.066 [arXiv:1205.5761 [nucl-ex]].

[16] The ATLAS collaboration [ATLAS Collaboration], ATLAS-CONF-2017-012.

[17] V. Khachatryan et al. [CMS Collaboration], JHEP 1704, 039 (2017) doi:10.1007/JHEP04(2017)039 [arXiv:1611.01664 [nucl-ex]]. 
[18] A. M. Sirunyan et al. [CMS Collaboration], Phys. Lett. B 776, 195 (2018) doi:10.1016/j.physletb.2017.11.041 [arXiv:1702.00630 [hep-ex]].

[19] S. Acharya et al. [ALICE Collaboration], JHEP 1811, 013 (2018) doi:10.1007/JHEP11(2018)013 [arXiv:1802.09145 [nucl-ex]].

[20] J. Adam et al. [ALICE Collaboration], Phys. Rev. Lett. 116, no. 13, 132302 (2016) doi:10.1103/PhysRevLett.116.132302 [arXiv:1602.01119 [nucl-ex]].

[21] J. Noronha-Hostler, B. Betz, J. Noronha and M. Gyulassy, Phys. Rev. Lett. 116, no. 25, 252301 (2016) doi:10.1103/PhysRevLett.116.252301 [arXiv:1602.03788 [nucl-th]].

[22] M. Gyulassy, P. Levai, J. Liao, S. Shi, F. Yuan and X. N. Wang, Nucl. Phys. A 982, 627 (2019) doi:10.1016/j.nuclphysa.2018.08.038 [arXiv:1808.03238 [hep-ph]]. 